\title{
Eyes absent homologue 2 predicts a favorable prognosis in colorectal cancer
}

This article was published in the following Dove Press journal:

OncoTargets and Therapy

\begin{abstract}
Jie Zheng ${ }^{1,2, *}$
Fuao $\mathrm{Cao}^{3, *}$

Xiaopei Huang ${ }^{4, *}$

Kuvaneshan Ramen ${ }^{2}$

Xiaowen $\mathrm{Xu}^{3}$

Yan Zhu ${ }^{5}$

Wenjun Chang $^{4}$

Yunfeng Shan ${ }^{2}$

Aizhen Guo'

'Department of General Medicine, Yangpu Hosptial, Tongji University School of Medicine, Shanghai 200090, People's Republic of China; ${ }^{2}$ Department of General Surgery, First Affiliated Hospital, Wenzhou Medical University, Zhejiang 3250I5, People's Republic of China; ${ }^{3}$ Department of Colorectal Surgery, Changhai Hospital, Second Military Medical University, Shanghai 200433, People's Republic of China; ${ }^{4}$ Department of Environmental Hygiene, Second Military Medical University, Shanghai 200433, People's Republic of China; ${ }^{5}$ Department of Pathology, Changhai Hospital, Second Military Medical University, Shanghai 200433, People's Republic of China
\end{abstract}

*These authors contributed equally to this work

Correspondence: Aizhen Guo Department of General Medicine, Yangpu Hosptial, Tongji University School of Medicine, 450 Tengyue Road, Shanghai 200090, People's Republic of China Tel +86 02। 6505 II 22 I223 24

Email cwjcwj1976@smmu.edu.cn

Yunfeng Shan

Department of General Surgery, First Affiliated Hospital, Wenzhou Medical University, 2 Fuxue Road, Wenzhou, Zhejiang 3250I5,

People's Republic of China

Tel +865775557 8027

Email shanyf@yahoo.com
Purpose: Eyes absent homologue 2 (EYA2), which functions as a transcription activator and phosphatase, plays an important role in several types of cancer. However, the impact of EYA2 in colorectal cancer (CRC) remains elusive.

Patients and methods: We evaluated the significance of EYA2 expression in the development and progression of CRC in a large cohort, including 922 CRC cases. EYA2 protein expression was determined via immunohistochemistry in colorectal tissues. The correlation between EYA2 expression and CRC occurrence was investigated in tumor tissue and the adjacent normal tissues. Factors contributing to CRC prognosis were evaluated using Kaplan-Meier and Cox model analyses.

Results: EYA2 expression was progressively lower in the adjacent normal tissue, adenomas, primary tumor and the metastatic CRC (all $P<0.05$ ). Furthermore, EYA2 expression had significant associations with disease stage, differentiation grade, and number of resected lymph nodes (all $P<0.001$ ). Compared with patients with EYA2-high tumors, those with EYA2-low tumors had shorter disease-free survival (hazard ratio [HR], 2.347; 95\% CI, 1.665-3.308) and disease-specific survival (HR, 3.560; 95\% CI, 2.055-6.167) in multivariate Cox analysis, after adjusting confounding factors such as tumor-node-metastasis stage and grade. In particular, patients with stage II or III EYA2-low CRC might be harmed by postoperative chemotherapy.

Conclusion: EYA2 expression was generally reduced in CRC. Higher EYA2 expression can predict a more favorable prognosis for CRC.

Keywords: colorectal cancer, EYA2, immunohistochemistry, prognosis, chemotherapy

\section{Introduction}

Colorectal cancer (CRC) is the third most commonly diagnosed malignancy worldwide. ${ }^{1}$ Surgical resection, supplemented with chemotherapy in certain circumstances, remains the primary curative method for localized and regional CRC. Despite the effectiveness of the regimen, the rate of relapse or metastasis after surgery remains high $(\sim 30 \%-50 \%)$, which is a major contributor of CRC-related death. ${ }^{2}$ Presently, the response of CRC patients to the current established regimen is quite heterogenous, even among patients with the same tumor-node-metastasis (TNM) stages. ${ }^{3}$ Moreover, $10 \%-20 \%$ of patients with stage II CRC and $30 \%-40 \%$ of those with stage III CRC eventually develop recurrence after therapeutic intervention. Additionally, adjuvant chemotherapy is beneficial to patients who are more likely to relapse after surgery but harmful to those who may not relapse. Clearly, the critical point in the current clinical situation is to identify those patients with a higher risk for relapse using effective biomarkers to ensure purposeful treatment. However, thus far, microsatellite instability (MSI) is the 
only validated marker for determining CRC prognosis and the appropriate chemotherapy. ${ }^{4,5}$ Therefore, new biomarkers are urgently required.

Eyes absent homologue 2 (EYA2) is a member of the eyes absent (EYA) family, functioning both as a transcriptional coactivator in conjunction with SIX family proteins (vertebrate homologues of the Drosophila gene sine oculis) and as a tyrosine phosphatase. ${ }^{6-10}$ The related biological processes controlled by this gene include the regulation of cell death and/or differentiation and DNA damage repair. ${ }^{10,11}$ Recently, the role of EYA2 in cancer has gained a lot of attention. Studies have shown that EYA2 is elevated in various cancer types including epithelial ovarian cancer, ${ }^{12}$ lung cancer, ${ }^{13,14}$ breast cancer, ${ }^{15}$ and cervical cancer. ${ }^{16}$ These studies have also shown that overexpression of EYA2 facilitates tumor growth and distant metastasis in vitro and/or in vivo models. In addition, the high expression of the EYA2 protein was found to be significantly associated with short overall survival in advanced ovarian cancer $^{12}$ and significantly associated with lung metastasis in breast cancer. ${ }^{15}$ Collectively, these findings demonstrate that EYA2 plays an oncogenic role in the mentioned cancer types. However, in another study, reduced expression of EYA2 in pancreatic adenocarcinoma promoted the growth and metastases of tumor cells, ${ }^{17}$ which is antagonistic to the function of EYA2 as an oncogene. Therefore, the role of EYA2 in malignancies remains controversial. Thus far, no study has described the biological function and clinical significance of EYA2 in CRC. Some evidence from previous studies indicates that the expression of EYA2 in CRC cell lines may be lower than that in cell lines from other cancer types, ${ }^{12}$ and methylation of EYA2 frequently occurs in CRC. ${ }^{18}$ The evidence involving CRC suggests that EYA2 may have different biological functions and clinical significances. Therefore, a more thorough investigation of the significance of EYA2 in CRC is required.

Herein, we conducted an immunohistochemical examination of EYA2 on tissue microarrays (TMAs), constructed using tissue specimens obtained from 922 CRC patients, to investigate the expression pattern of EYA2 among different colorectal tissues and the association between patient survival and EYA2 expression. The results showed that EYA2 may be a promising biomarker for the stratification of prognosis and the prediction of effective responses to chemotherapy in CRC patients.

\section{Patients and methods}

\section{Bioinformatics analysis of EYA2 in CRC}

The microarray expression profiles of three data sets (GSE62294, GSE37364, and GSE41258) related to colorectal tissues were downloaded from the Gene Expression Omnibus (GEO) database (http://www.ncbi.nlm.nih.gov/geo/). The pathological types and sample sizes of each data set are listed in Figure 1A. The expression profiles of EYA2 in the related specimens were extracted from the processed data and then compared among different pathological types of colorectal tissues via independent sample $t$-tests. In addition, the expression patterns of EYA1, EYA3, and EYA4 in CRC were analyzed.

\section{Patient characteristics}

A total of 1,022 colorectal tissue specimens, donated by 922 patients who underwent surgical treatment at Changhai Hospital, Second Military Medical University (Shanghai, People's Republic of China), between January 2001 and November 2011, were used to develop a series of tissue microarrays (TMAs) via a commercial company (Outdo Biotech, Shanghai, People's Republic of China) as reported previously. ${ }^{19}$ One core was used from each sample, with a core diameter of $1.2 \mathrm{~mm}$. The TMAs contained 68 normal colorectal tissues, 32 colorectal adenomas, 906 primary CRC samples, and 16 metastatic CRC samples. All the patients were selected by pathological diagnosis and staged according to the American Joint Committee on Cancer Staging System (seventh edition). The baseline characteristics of the 906 donors with primary CRC are summarized in Table 1, including information on age, sex, disease location, TNM stage, histological grade, number of resected lymph nodes at surgery, standard postoperative chemotherapy (FOLFOX regimen), serum carcinoembryonic antigen (CEA), and carbohydrate antigen 199 (CA199). This study was approved by the Institutional Review Board of Changhai Hospital. All individual participants signed a written informed consent, giving agreement for the trial to use biological material for research purposes.

\section{Immunohistochemistry (IHC)}

IHC was carried out using $4 \mu \mathrm{m}$ thick sections in the Department of Environment Hygiene, Second Military Medical University. Rabbit antihuman EYA2 polyclonal antibody (1:500, HPA027024; Sigma-Aldrich Co., St Louis, MO, USA) was used based on the manufacturer's instructions. Antibody specificity was previously verified in the Human Protein Atlas (http://www.proteinatlas.org/). Notably, the epitope antigens were retrieved using citrate buffer ( $\mathrm{pH}$ 6.0) at $100^{\circ} \mathrm{C}$ in a pressure cooker (KEDA, Shanxi, People's Republic of China) for 5 minutes. Nonspecific binding was blocked through incubation with $10 \%$ normal goat serum (SL038; Solarbio, Beijing, People's Republic of China) at room temperature for 30 minutes. Subsequently, the tissue 
A
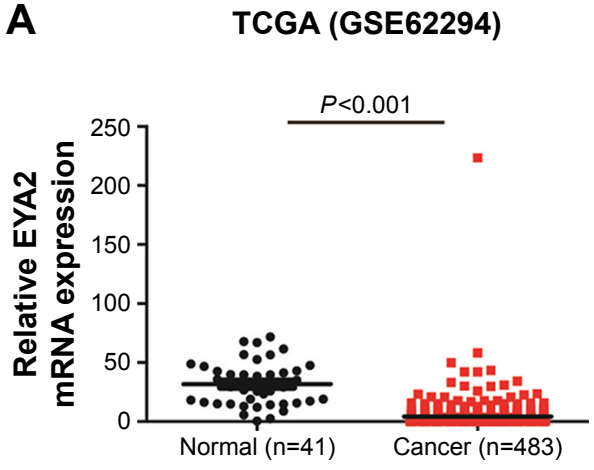

GSE37364

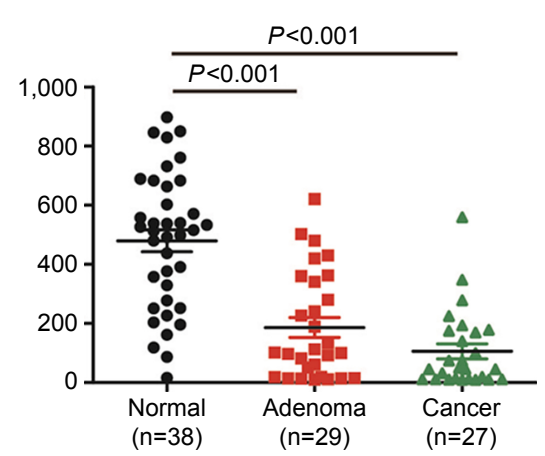

GSE41258

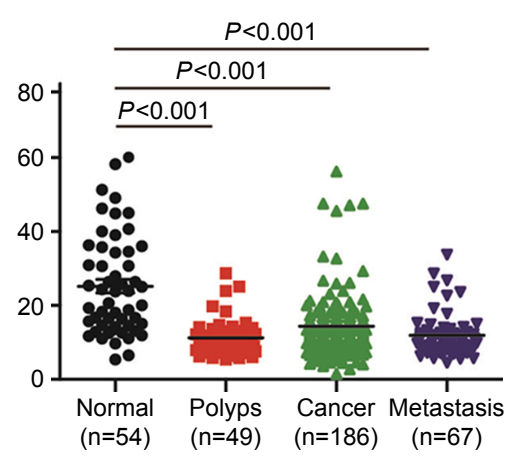

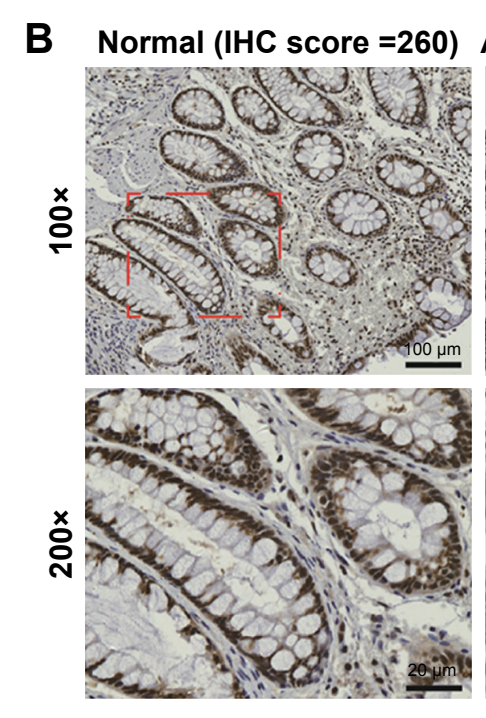

C

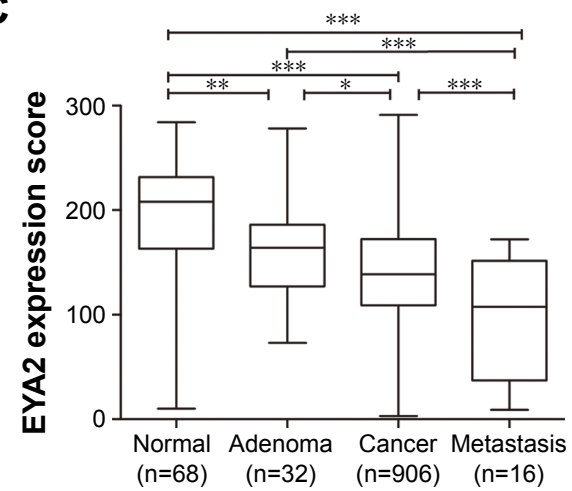

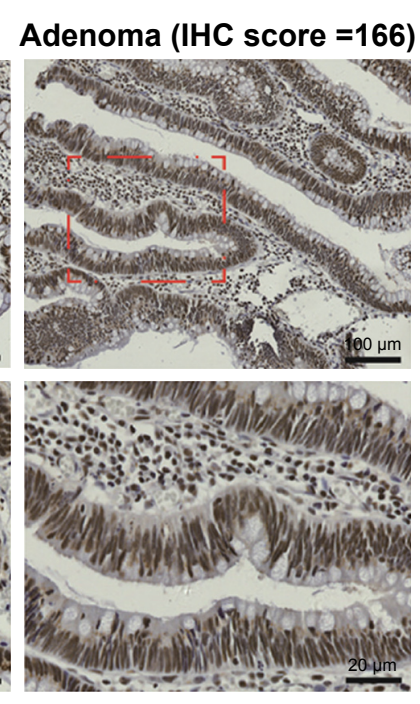

D

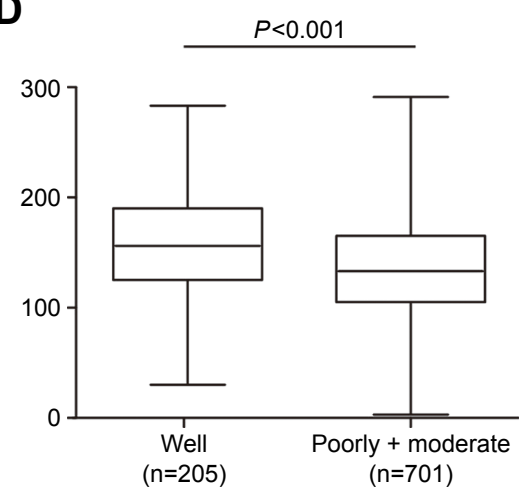

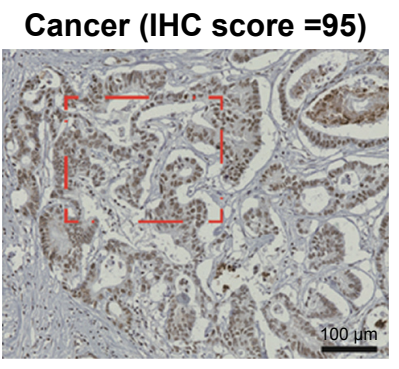

Metastasis (IHC score $=17$ )
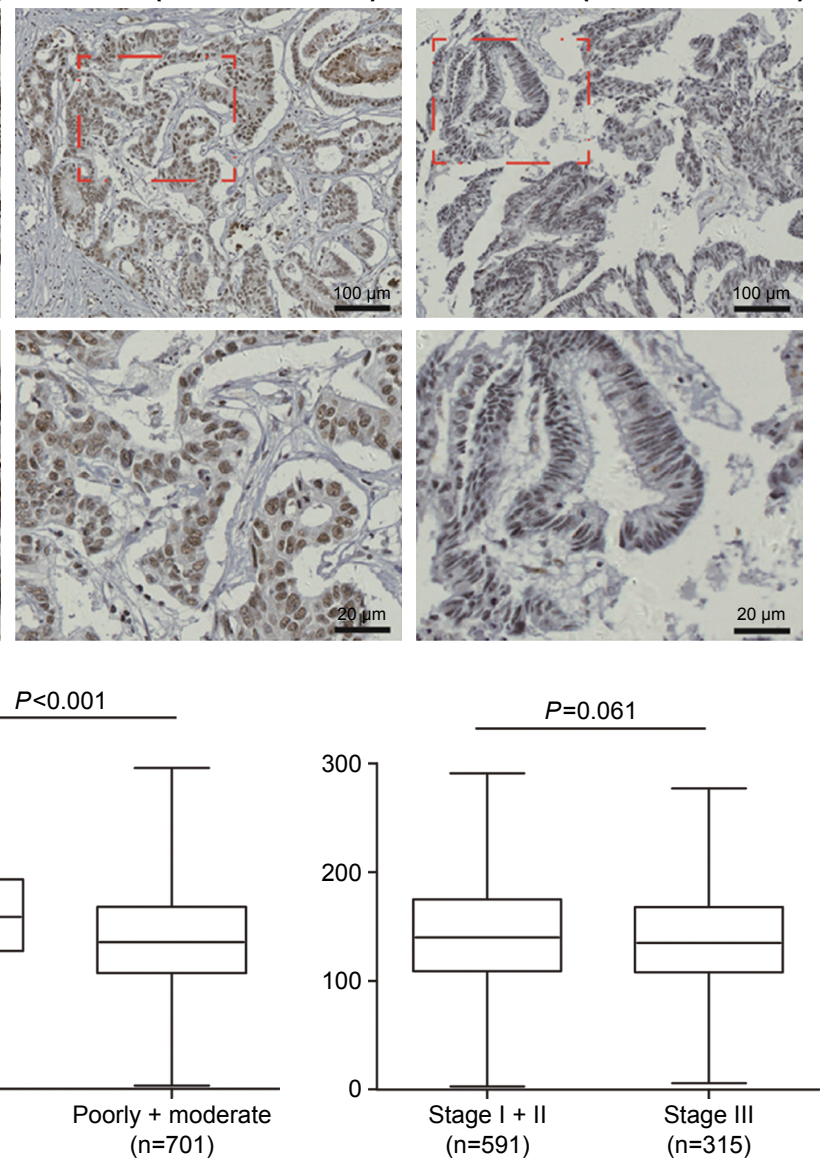

Figure I Expression of EYA2 is reduced in CRC.

Notes: (A) Bioinformatics analyses of EYA2 mRNA expression between cancer and cancer-related specimens in three datasets downloaded from GEO database. (B) Representative nuclear EYA2 staining in colorectal tissues. Bars, 100 or $20 \mu \mathrm{m}$. (C) Comparison of IHC H-score among different colorectal pathological tissues or between different TNM stages or between different grades. $* P<0.05$; $* * P<0.0$; $* * * P<0.00$ I. (D) Comparison of IHC H-score between different TNM stages or between different grades.

Abbreviations: CRC, colorectal cancer; EYA2, eyes absent homologue 2; GEO, Gene Expression Omnibus; IHC, immunohistochemistry; TNM, tumor-node-metastasis.

slides were incubated with the rabbit antihuman EYA2 polyclonal antibody at $4^{\circ} \mathrm{C}$ overnight and then incubated with an HRP-Polymer Anti-Rabbit IHC Kit (KIT-5920; Maxvision, Fuzhou, People's Republic of China). All the TMAs were stained simultaneously by a technician to eliminate interassay variation.
Quantitative evaluation of immunostaining The stained TMA slides were observed under bright-field microscopy at a resolution of $\times 20$ (digital scanning via Aperio Scanscope v12.1; Aperio Technologies, Vista, CA, USA) to allow quantitative image analysis $(\times 200)$. The EYA2 protein was quantitated using the $H$-score method as previously 
Table I Associations of epithelial nuclear EYA2 expression with demographic and clinical variables of 906 CRC patients

\begin{tabular}{|c|c|c|c|c|}
\hline \multirow[t]{2}{*}{ Characteristics } & \multirow[t]{2}{*}{$\begin{array}{l}\text { Total } \\
(n=906)\end{array}$} & \multicolumn{2}{|c|}{$\begin{array}{l}\text { Nuclear EYA2 } \\
\text { expression }\end{array}$} & \multirow[t]{2}{*}{$P$-value* } \\
\hline & & $\begin{array}{l}\text { Low } \\
(n=509)\end{array}$ & $\begin{array}{l}\text { High } \\
(n=397)\end{array}$ & \\
\hline Mean age \pm SD (year) & $60.9 \pm 12.5$ & $61.1 \pm 12.5$ & $60.6 \pm 12.5$ & $0.587 * *$ \\
\hline \multicolumn{4}{|l|}{ Sex, n (\%) } & 0.263 \\
\hline Male & $545(60.2)$ & $298(58.5)$ & $247(62.2)$ & \\
\hline Female & $361(39.8)$ & $211(41.5)$ & $150(37.8)$ & \\
\hline \multicolumn{4}{|c|}{ Disease location, n (\%) } & 0.139 \\
\hline Rectum & $502(55.4)$ & $293(57.6)$ & $209(52.6)$ & \\
\hline Colon & $404(44.6)$ & $216(42.4)$ & $188(47.4)$ & \\
\hline \multicolumn{4}{|c|}{ Differentiation grade, $n(\%)$} & $<0.001 * * *$ \\
\hline Well & $102(11.2)$ & $38(7.5)$ & $64(16.1)$ & \\
\hline Moderately & $749(82.7)$ & $448(88.0)$ & $301(75.8)$ & \\
\hline Poorly & $39(4.3)$ & $2 I(4 . I)$ & $18(4.5)$ & \\
\hline Missing & $16(1.8)$ & $2(0.4)$ & $14(3.5)$ & \\
\hline \multicolumn{4}{|c|}{ Resected lymph nodes, n (\%) } & $<0.001$ \\
\hline$<12$ & $205(22.6)$ & $18(10.6)$ & $187(25.4)$ & \\
\hline$\geq 12$ & $70 \mathrm{I}(77.4)$ & $152(89.4)$ & $549(74.6)$ & \\
\hline \multicolumn{4}{|l|}{ TNM stage, $n(\%)$} & $<0.00 I^{* * *}$ \\
\hline 1 & $|3|(\mid 4.4)$ & $88(17.3)$ & $43(10.8)$ & \\
\hline ॥ & $460(50.8)$ & $234(46.0)$ & $226(56.9)$ & \\
\hline III & $315(34.8)$ & 187 (36.7) & $128(32.2)$ & \\
\hline \multicolumn{4}{|l|}{ Chemotherapy, n (\%) } & 0.122 \\
\hline Yes & $673(74.3)$ & $368(72.3)$ & $305(76.8)$ & \\
\hline No & $233(25.7)$ & $|4|$ (27.7) & $92(23.2)$ & \\
\hline \multicolumn{4}{|l|}{ Serum CEA, $n(\%)$} & 0.606 \\
\hline$<5 \mathrm{ng} / \mathrm{mL}$ & $562(62.0)$ & $312(6 \mid .3)$ & $250(63.0)$ & \\
\hline$\geq 5 \mathrm{ng} / \mathrm{mL}$ & $344(38.0)$ & 197 (38.7) & $147(37.0)$ & \\
\hline \multicolumn{4}{|l|}{ Serum CAI9-9, n (\%) } & 0.647 \\
\hline$<37 \mathrm{U} / \mathrm{mL}$ & 775 (85.5) & $433(85.1)$ & $342(86.1)$ & \\
\hline$\geq 37 \mathrm{U} / \mathrm{mL}$ & $13 \mid(\mid 4.5)$ & $76(14.9)$ & $55(13.9)$ & \\
\hline
\end{tabular}

Notes: Missing values are excluded for all statistic tests. ${ }^{*} \chi^{2}$ test or Fisher's exact test. **Student's $t$-test. ***Mann-Whitney $U$ test (nonparametric).

Abbreviations: CA19-9, carbohydrate antigen 19-9; CEA, carcinoembryonic antigen; CRC, colorectal cancer; EYA2, eyes absent homologue 2; TNM, tumor-nodemetastasis.

described. ${ }^{20}$ Immunostaining for EYA2 was assessed by determining the percentage of epithelial or mesenchymal cells showing negative $(0)$, weak $(1+)$, moderate $(2+)$, and strong $(3+)$ nuclear staining. The total number of cells in each field and the number of cells stained at each intensity were counted. $H$-scores were calculated by multiplying the average percentage of positively stained cells and the corresponding intensity of staining (yielding a score ranged from 0 to 300). Two independent investigators with no prior knowledge of the clinicopathological information carried out the staining assessment. The interobserver differences were averaged.

\section{Follow-up and patient subgroups}

Follow-up information for the 906 patients with stages I-III CRC was collected following a standard procedure as previously described. ${ }^{19}$ The primary outcome of interest was disease-specific survival (DSS), which was defined as the number of months from the date of undergoing surgery to the date of death due to CRC. Disease-free survival (DFS) was measured in months from the date of undergoing surgery to the date of the first relapse. An optimal cutoff value for epithelial EYA2 IHC scores was selected to divide patients into the subgroups of high and low EYA2 expressions. This was carried out using the package maxstat in $\mathrm{R}$ 3.2.0 (www.r-project.org), which can effectively discriminate survival differences.

\section{Statistical analyses}

The patient's clinicopathological characteristics were summarized descriptively and tested using two-sample Student $t$-tests (continuous variables) and Pearson's Chi-square tests (categorical variables). Wilcoxon-Mann-Whitney tests were used to analyze the TNM stage and histological grade. The DFS and DSS of subgroups were compared to determine survival outcomes using Kaplan-Meier curves and then further analyzed with the log-rank test to observe the significance. All possible prognostic factors were determined using a univariate Cox proportional hazards model; subsequently, the meaningful prognostic factors (defined as those with $P<0.05$ in univariate analysis) were further assessed in a multivariate analysis. Interaction between EYA2 expression status and chemotherapy was evaluated using Cox proportional hazards models with a $2 \times 2$ factorial design. ${ }^{21}$ All statistical analyses were conducted using SPSS V.19.0 for Windows (IBM Corporation, Armonk, NY, USA). Significance was set at $P<0.05$ for two-sided tests.

\section{Results}

\section{Expression of EYA2 is reduced in CRC}

To explore the potential significance of EYA2 in CRC, we initially compared the expression pattern of EYA2 between $\mathrm{CRC}$ tissue and the adjacent normal tissue in a bioinformatics analysis. We found that the level of EYA2 mRNA in CRC was consistently significantly lower than that in the adjacent normal tissues in the three datasets obtained from the GEO database (all $P<0.01$; Figure 1A). However, expression did not differ significantly across the three datasets for EYA1, EYA3, or EYA4 (Figure S1). Subsequently, the expression pattern of EYA2 was investigated via IHC examination in 906 CRC and 68 adjacent normal tissue specimens obtained from patients at Changhai Hospital. The immunostaining for EYA2 showed positive results predominantly in the nuclei of colorectal epithelial cells and some mesenchymal cells 
(Figure 1B). Results from the independent Student's $t$-test showed that the expression of nuclear EYA2 protein in epithelial cells was significantly lower in CRC tissue than that in the corresponding adjacent normal tissue specimens $(P<0.001$; Figure 1C). Notably, we found that nuclear EYA2 expression in epithelial cells was progressively lower in adjacent normal tissues, adenomas, and primary cancer and metastatic CRC (all $P<0.05$; Figure 1B and $\mathrm{C}$ ), which indicated that reduced EYA2 may be associated with CRC progression. However, negative or weak EYA2 expression with low frequency $(<5 \%$ specimens) was detected in mesenchymal cells surrounding epithelial cancer cells (Figure 1B).

\section{Associations between EYA2 expression and clinicopathological characteristics in patients with CRC}

In further investigations, we compared epithelial EYA2 protein levels with the clinicopathological characteristics of patients with CRC. There were significant differences between EYA2 expression and differentiation grade $(P<0.001)$, as well as marginally significant differences $(P=0.061)$ between EYA2 expression and TNM stages when EYA2 expression was analyzed as a continuous variable (Figure 1D). Next, we classified the 906 patients with stages I-III CRC into EYA2-low or EYA2-high subgroups using the optimal cutoff value (IHC score $=145$ ) identified via the maxstat $R$ package. No significant associations were found between EYA2 expression and age, sex, disease location, serum CEA level, serum CA199 level, or postoperative adjuvant chemotherapy (all $P>0.05$ ). However, EYA2 expression differed significantly according to TNM stage, grade, and number of resected lymph nodes (all $P<0.001$; Table 1), which indicated that EYA2 may be involved in the aggressiveness of CRC. Therefore, we proceeded to assess the association between EYA2 expression and survival outcomes using the IHC data.

\section{Low expression of EYA2 protein predicted a poor prognosis in CRC}

As presented in Table 2, a univariate Cox regression analysis based on the Cox proportional hazards model was applied to determine the hazard ratio (HR) of clinicopathological characteristics according to the follow-up DFS and DSS of CRC patients. Poor histological grade, advanced TNM stage, a higher number of resected lymph nodes, adjuvant chemotherapy, high level of serum CEA and serum CA199, and low EYA2 expression predicted poor DSS and/or shorter DFS for CRC (Table 2). In the multivariate

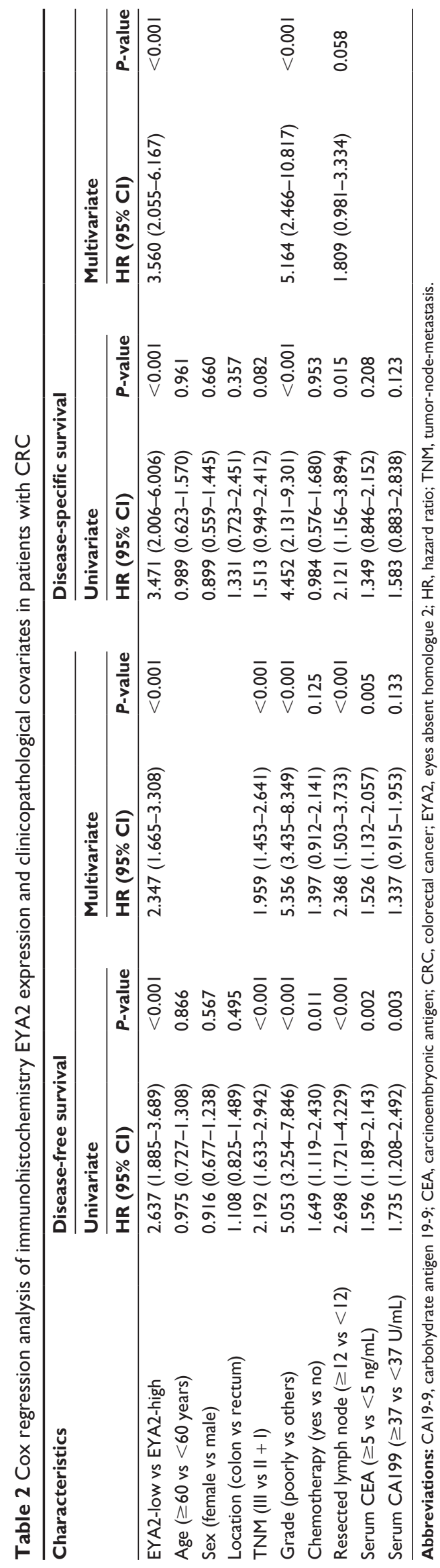


analysis, the HR for CRC relapse among patients with EYA2-low tumors versus EYA2-high tumors was 2.347 (95\% CI, 1.665-3.308; $P<0.001)$ after adjusting variables such as tumor stage, grade, chemotherapy, number of resected lymph nodes, and serum CEA and CA199 levels. The level of EYA2 expression was also an independent risk factor for DSS with a HR of 3.560 (95\% CI, 2.055-6.167; $P<0.001)$. Kaplan-Meier analysis revealed that patients with EYA2-low tumors (IHC score $\leq 145$ ) had significantly shorter DFS and DSS when compared with those with EYA2-high tumors (IHC score $>145$; all $P<0.001$; Figure 2). Notably, patients with stage II and those with stage III CRC with EYA2-low tumors were consistently associated with worse DFS and DSS than those with EYA2high tumors (all $P<0.05$ ); this trend was not observed for patients with stage I CRC (all $P>0.05$ ).

\section{Low EYA2 protein expression is associated with potential harm from chemotherapy}

To evaluate whether patients with EYA2-high tumors might benefit from adjuvant chemotherapy, we investigated the association between EYA2 expression levels and survival outcomes among patients who did and those who did not receive adjuvant chemotherapy. Since all the patients with stage I CRC did not receive adjuvant chemotherapy, we focused on the patients with stage II and stage III CRC (Table S1). We observed that high EYA2 protein expression was associated with a longer DFS and DSS than that observed with low EYA2 protein expression in patients with stage II as well as those with stage III CRC who received adjuvant chemotherapy (all $P<0.05$; Figure 3 ); this trend was not observed for patients who did not receive adjuvant chemotherapy (all $P>0.05$; Figure 3 ). Additionally, when we analyzed the subgroup of patients who had stage II disease and EYA2-low protein expression, we observed that use of chemotherapy had a clear association with reduced survival outcomes (Figure 3). Furthermore, when we analyzed patients with stage II disease, testing the interaction between EYA2 expression and chemotherapy status revealed that there was a significant interaction $(P=0.041)$ for DFS. Therefore, our results indicated that patients with low EYA2 expression might be harmed by adjuvant chemotherapy.

\section{Discussion}

In this study, we initially compared the expression pattern of EYA2 between CRC tissue and adjacent normal tissue
A

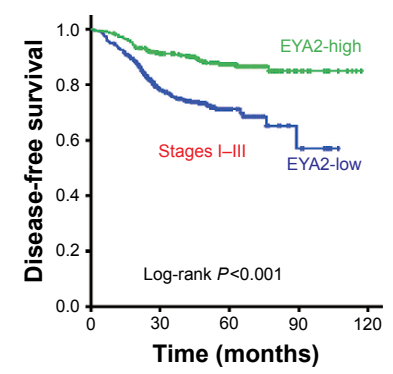

Number of

tients at risk

$\begin{array}{rlllll}\text { EYA2-low } & 509 & 300 & 66 & 7 & 0 \\ \text { EYA2-high } & 397 & 317 & 145 & 21 & 0\end{array}$

B

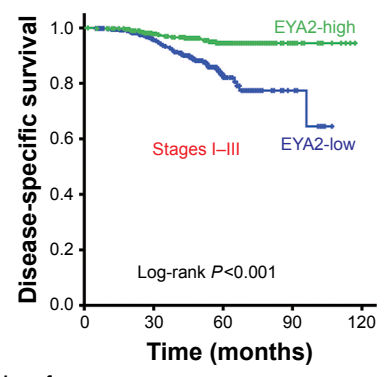

Number of
patients at risk

$\begin{array}{llllll}\text { EYA2-low } & 509 & 339 & 76 & 8 & 0\end{array}$

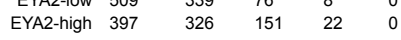
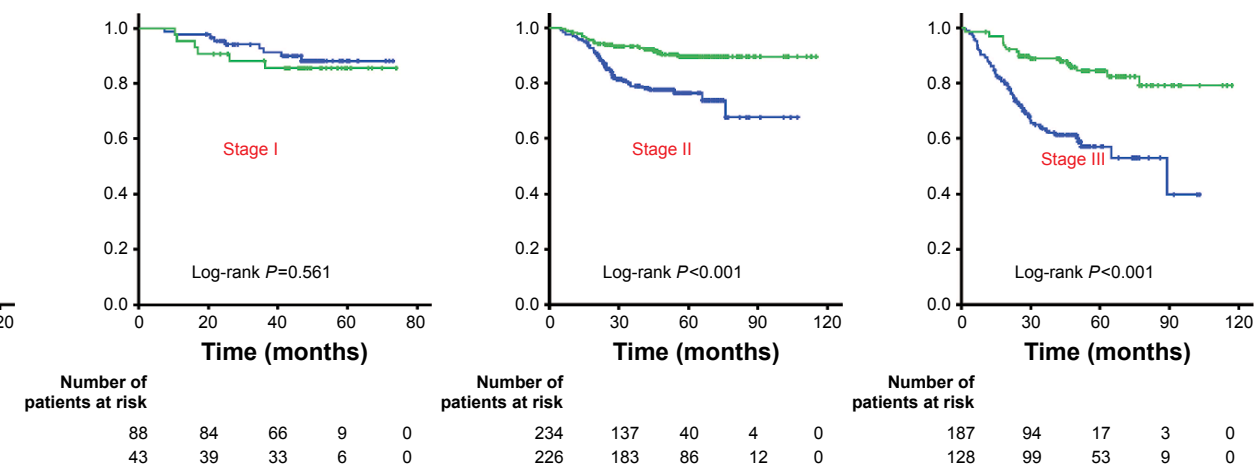

Number of
patients at risk
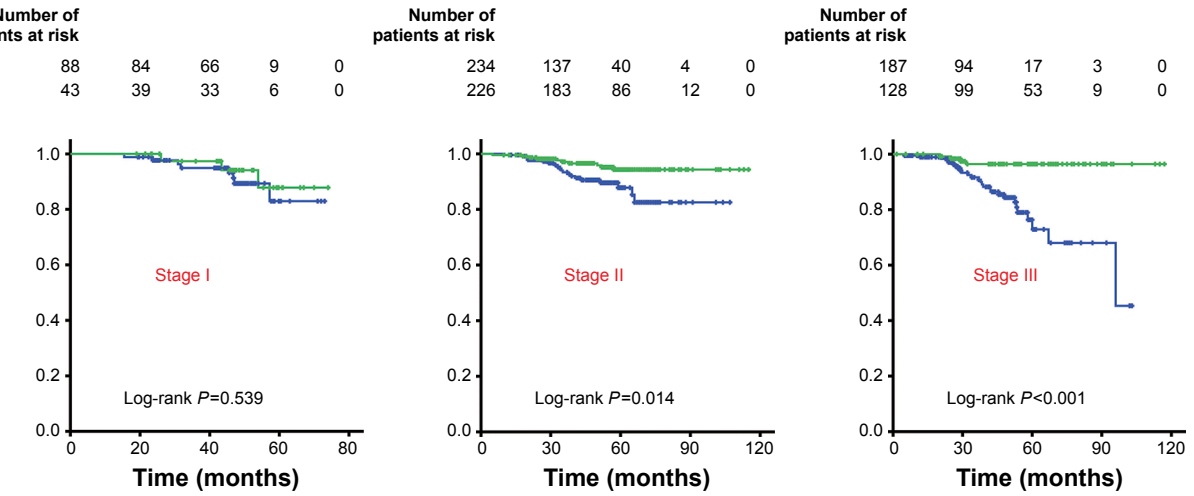

Number of
patients at risk
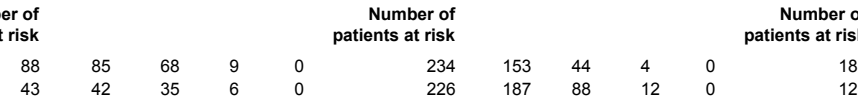

Figure 2 Low EYA2 protein predicted a poor prognosis in patients with CRC.

Notes: (A) Associations between EYA2 expression and DFS in the patient subgroups with different stage tumors. (B) Associations between EYA2 expression and OS in the patient subgroups with diffrent stage tumors. Patients with stages I-III, stage I, stage II, or stage III tumor were dichotomized into the subgroups with high- or low-EYA2 protein expression according to EYA2 IHC score (cutoff value $=145$ ). Kaplan-Meier survival curves reveal DFS and DSS in patients with each TNM stage CRC. P-values are from Kaplan-Meier analysis with log-rank test.

Abbreviations: CRC, colorectal cancer; DFS, disease-free survival; DSS, disease-specific survival; EYA2, eyes absent homologue 2; IHC, immunohistochemistry; TNM, tumornode-metastasis. 

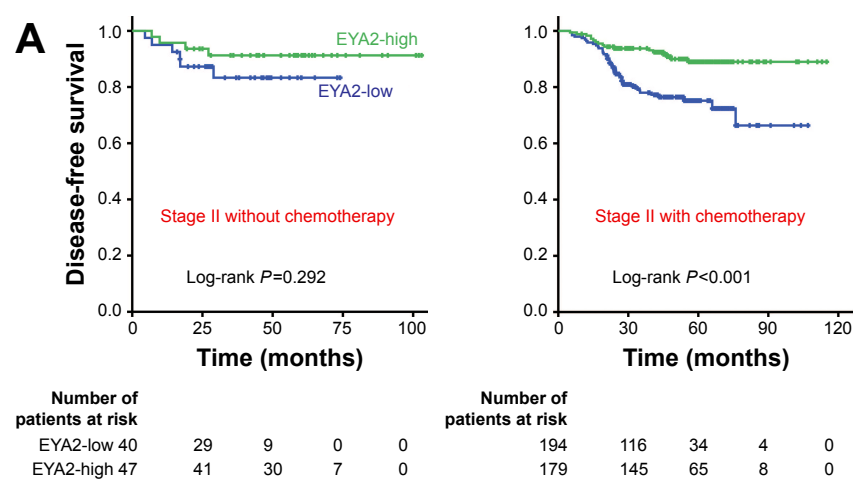

B
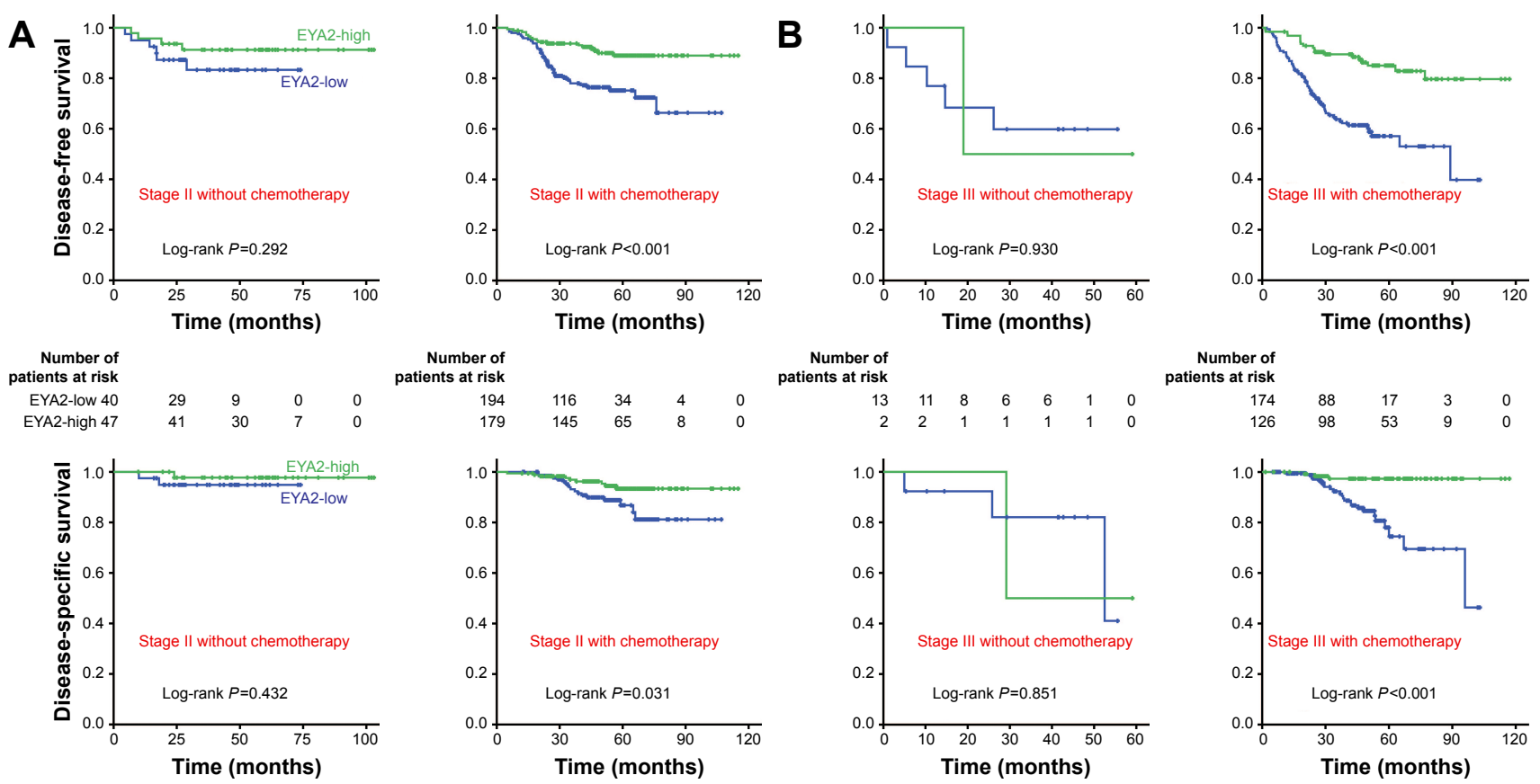

Number of

patients at risk

EYA2-low 40

EYA2-high 47

$\begin{array}{llll}42 & 31 & 7 & 0\end{array}$
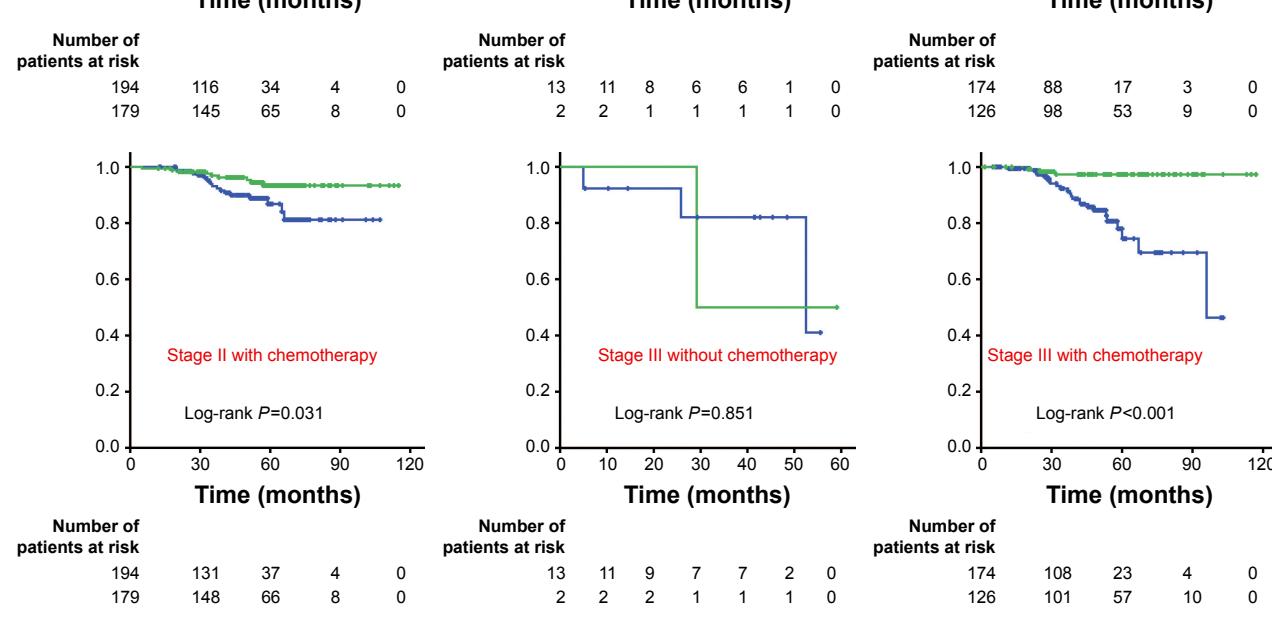

Figure 3 High EYA2 protein could predict a benefit of adjuvant chemotherapy in CRC patients.

Notes: (A) Associations between EYA2 expression and patients outcomes in the stage Il patients with or without chemotherapy. (B) Associations between EYA2 expression and patients outcomes in the stage III patients with or without chemotherapy. Kaplan-Meier survival curves of EYA2-high (green line) and EYA2-low (blue line) patients with stage II and stage III CRC who did or did not receive postoperative adjuvant chemotherapy are shown. P-values are from Kaplan-Meier analysis with log-rank test.

Abbreviations: CRC, colorectal cancer; EYA2, eyes absent homologue 2.

using publicly available expression profiles; we found that the transcriptional levels of EYA2 in CRC were consistently lower than those in the adjacent normal tissues in three data sets, including the The Cancer Genome Atlas (TCGA) CRC microarray dataset. The IHC examination of EYA2 on TMAs showed that the EYA2 protein was mainly distributed in the nuclei of colorectal epithelial cells and the nuclei of mesenchymal cells surrounding normal epithelial cells. Furthermore, EYA2 IHC confirmed that CRC tissues had lower epithelial EYA2 protein expression than normal tissues in the Changhai cohort. Interestingly, the expression of epithelial EYA2 in colorectal polyps was also significantly lower than that observed in normal tissue, which suggested that the reduction in epithelial EYA2 was associated with CRC occurrence and may be an early event in the development of CRC.

EYA2 has been reported as an intrinsically cytosolic protein that is translocated to the nucleus by conjunction with the members of SIX family proteins. ${ }^{7,8}$ Previous studies have reported that the EYA2 protein can be detected via IHC in both the nucleus and cytoplasm of epithelial cells in ovarian cancer and non-small-cell lung cancer ${ }^{12,22}$ but could be observed only in the nucleus of some primary pancreatic cancer. Moreover, the expression pattern of EYA2 in CRC is different from that in other cancer types, such as epithelial ovarian cancer, lung cancer, breast cancer, and cervical cancer, ${ }^{12-16,23}$ where EYA2 is significantly elevated in cancer lesions. Recently, the expression of EYA2 in pancreatic cancer has also been reported to be lower than that in the adjacent normal tissues. ${ }^{17}$ Various expression patterns of EYA2 in different cancer types suggest that the function of EYA2 may be organ or tissue specific. ${ }^{7}$ In our study, a decreasing trend in EYA2 expression in epithelial nuclei was detected but rarely in the cytoplasm, during the progress of CRC. These data also suggest that EYA2 has organ-specific functions, presumably depending in part on its subcellular localization and focusing more on the complicated interactions of its double roles of a phosphatase and transcription coactivator during differentiation and cell cycle progression. ${ }^{10}$

In the analysis of the associations between EYA2 expression and the clinicopathological characteristics of patients with CRC, we found that low EYA2 expression was related to advanced TNM stages and poor differentiation. Since TNM stages and differentiation grades are usually associated with CRC prognosis, we proceeded to classify patients into EYA2-high or EYA2-low CRC subgroups according to the optimal EYA2 IHC score cutoff. We found that the patients with EYA2-low tumors had a shorter DFS and DSS than those 
with EYA2-high tumors. In the multivariate Cox analysis, low EYA2 expression was an independent risk factor for CRC prognosis, in the presence of confounding factors such as TNM stage and grade. These results demonstrated that EYA2 expression may be a potential supplementation when TNM stage served as a criterion for prognostic stratification. After adjusting for TNM stage as a confounding factor, we found that the expression of the EYA2 protein was significantly associated with DFS and DSS in stage II and stage III CRC. Obviously, the prognostic significance of EYA2 in CRC is completely different from that observed in ovarian cancer ${ }^{12}$ but is consistent with the results for pancreatic cancer. ${ }^{17}$ Nevertheless, because information on some characteristics was incomplete or unavailable (eg, for MSI, KRAS or BRAF mutation, and other factors), the prognostic significance of EYA2 should be validated in larger prospective cohorts in the future.

Adjuvant chemotherapy (FOLFOX/CapeOX regimen) is considered as a standard therapy for patients with stage III and a proportion of patients with stage II CRC after curative resection. However, the survival benefit observed from the regimen was primarily limited to patients with stage III disease; thus, the use of adjuvant chemotherapy for patients with stage II CRC remains controversial. ${ }^{24,25}$ Therefore, we further analyzed the interaction between EYA2 expression and adjuvant chemotherapy in patients with stage II or stage III CRC and found that the different expression levels of the EYA2 protein had no association with DFS or DSS among patients who did not receive adjuvant chemotherapy. However, a significant difference in survival outcomes was identified between patients with stage II/III EYA2-high CRC and those with stage II/III EYA2-low CRC who received adjuvant chemotherapy. Interestingly, in a subgroup analysis of patients who had stage II disease and EYA2-low expression, we found that patients with chemotherapy had clearly reduced survival outcomes. This indicated that EYA2-low patients might be harmed by chemotherapy. Furthermore, the results of an interaction analysis implied that the reduction in survival outcomes may have been associated with the combination of reduced EYA2 expression and chemotherapy.

Regarding molecular mechanisms, it has been reported that EYA2 is a transcriptional activator, the activity of which depends on SIX family factors. ${ }^{6-10,12}$ Interestingly, SIX1-5 were detected in most ovarian and breast cancer cell lines but not in colon cancer cells, ${ }^{12}$ which indicates that the function of EYA2 as a transcriptional activator may be lost in colon cancer. EYA2 also has phosphatase activity and can reduce the phosphorylation of some key proteins. Recent studies have revealed that reduced EYA2 can promote TGFBR2 phosphorylation in pancreatic cancer cells ${ }^{17}$ and generate H2A.X ${ }^{\text {Tyr39 }}$ phosphorylation in colon cancer cells. ${ }^{26}$ For both of them, TGFBR2 phosphorylation can promote cancer aggressiveness via acting epithelial-mesenchymal transition (EMT) signaling and H2A. $\mathrm{X}^{\mathrm{Ty} 39}$ phosphorylation is a prerequisite of $\gamma$-H2A.X formation, which is a very important inducer for efficient repair of DNA double-strand breaks. ${ }^{26}$ It is also well known that increased DNA repair is usually associated with the development of resistance to chemotherapy in multiple cancer types. Moreover, some chemotherapy drugs (such as oxaliplatin) can also activate EMT signaling, especially in resistant cells. ${ }^{27,28}$ Therefore, it is possible that reduced EYA2 promotes CRC progression and generates unfavorable outcomes in the presence of chemotherapy via a complex signaling mechanism, such as enhancing TGF- $\beta$ signaling and/or DNA repair. However, this suggestion is speculative and little concrete evidence is available regarding the underlying mechanisms.

\section{Conclusion}

In this study, we systematically investigated the clinical significance of EYA2 expression in CRC and provided important evidence that EYA2 expression is downregulated in primary and metastatic CRC. Notably, we found that low EYA2 expression was significantly correlated with unfavorable DFS and DSS in patients with CRC, as well as with a survival reduction in CRC patients who received postoperative chemotherapy. Although our study has revealed that EYA2 could be a clinically promising biomarker in CRC, the molecular mechanisms behind the relationship between EYA2 and CRC remain uncertain and should be investigated further. Future studies should elucidate the roles that EYA2 plays in both the occurrence and progression of CRC.

\section{Acknowledgment}

This study was supported by grants from the National Natural Science Foundation of China (81402005 to FC and 81372671 and 81572451 to $\mathrm{WC}$ ).

\section{Disclosure}

The authors report no conflicts of interest in this work.

\section{References}

1. Torre LA, Bray F, Siegel RL, Ferlay J, Lortet-Tieulent J, Jemal A. Global cancer statistics, 2012. CA Cancer J Clin. 2015;65(2):87-108.

2. Schmoll HJ, Van Cutsem E, Stein A, et al. ESMO Consensus Guidelines for management of patients with colon and rectal cancer. A personalized approach to clinical decision making. Ann Oncol. 2012;23(10): $2479-2516$. 
3. Nagtegaal ID, Quirke P, Schmoll HJ. Has the new TNM classification for colorectal cancer improved care? Nat Rev Clin Oncol. 2011;9(2): $119-123$.

4. Hemminki A, Mecklin JP, Järvinen H, Aaltonen LA, Joensuu H. Microsatellite instability is a favorable prognostic indicator in patients with colorectal cancer receiving chemotherapy. Gastroenterology. 2000;119(4):921-928.

5. Popat S, Hubner R, Houlston RS. Systematic review of microsatellite instability and colorectal cancer prognosis. J Clin Oncol. 2005;23(3): 609-618.

6. Li X, Oghi KA, Zhang J, et al. Eya protein phosphatase activity regulates Six1-Dach-Eya transcriptional effects in mammalian organogenesis. Nature. 2003;426(6964):247-254.

7. Ohto H, Kamada S, Tago K, et al. Cooperation of six and eya in activation of their target genes through nuclear translocation of Eya. Mol Cell Biol. 1999;19(10):6815-6824.

8. Fan X, Brass LF, Poncz M, Spitz F, Maire P, Manning DR. The alpha subunits of $\mathrm{Gz}$ and $\mathrm{Gi}$ interact with the eyes absent transcription cofactor Eya2, preventing its interaction with the six class of homeodomaincontaining proteins. J Biol Chem. 2000;275(41):32129-32134.

9. Farabaugh SM, Micalizzi DS, Jedlicka P, Zhao R, Ford HL. Eya2 is required to mediate the pro-metastatic functions of Six 1 via the induction of TGF-beta signaling, epithelial-mesenchymal transition, and cancer stem cell properties. Oncogene. 2012;31(5):552-562.

10. Jemc J, Rebay I. The eyes absent family of phosphotyrosine phosphatases: properties and roles in developmental regulation of transcription. Annu Rev Biochem. 2007;76:513-538.

11. Krishnan N, Jeong DG, Jung SK, et al. Dephosphorylation of the C-terminal tyrosyl residue of the DNA damage-related histone H2A.X is mediated by the protein phosphatase eyes absent. J Biol Chem. 2009;284(24):16066-16070.

12. Zhang L, Yang N, Huang J, et al. Transcriptional coactivator Drosophila eyes absent homologue 2 is up-regulated in epithelial ovarian cancer and promotes tumor growth. Cancer Res. 2005;65(3):925-932.

13. Gao T, Zheng S, Li Q, et al. Aberrant hypomethylation and overexpression of the eyes absent homologue 2 suppresses tumor cell growth of human lung adenocarcinoma cells. Oncol Rep. 2015;34(5): 2333-2342.

14. Yuan Y, Zheng S, Li Q, et al. Overexpression of miR-30a in lung adenocarcinoma A549 cell line inhibits migration and invasion via targeting EYA2. Chin J Biochem Biophys. 2016;48(3):220-228.
15. Liang $\mathrm{Y}, \mathrm{Xu} \mathrm{X}$, Wang $\mathrm{T}$, et al. The EGFR/miR-338-3p/EYA2 axis controls breast tumor growth and lung metastasis. Cell Death Dis. 2017;8(7):e2928.

16. Bierkens M, Krijgsman O, Wilting SM, et al. Focal aberrations indicate EYA2 and hsa-miR-375 as oncogene and tumor suppressor in cervical carcinogenesis. Genes Chromosomes Cancer. 2013;52(1):56-68.

17. Vincent A, Hong SM, Hu C, et al. Epigenetic silencing of EYA2 in pancreatic adenocarcinomas promotes tumor growth. Oncotarget. 2014; 5(9):2575-2587.

18. Zou H, Harrington JJ, Shire AM, et al. Highly methylated genes in colorectal neoplasia: implications for screening. Cancer Epidemiol Biomarkers Prev. 2007;16(12):2686-2696.

19. Pan Y, Cao F, Guo A, et al. Endoplasmic reticulum ribosome-binding protein 1, RRBP1, promotes progression of colorectal cancer and predicts an unfavourable prognosis. Br J Cancer. 2015;113(5):763-772.

20. Detre S, Saclani Jotti G, Dowsett M. A “quickscore" method for immunohistochemical semiquantitation: validation for oestrogen receptor in breast carcinomas. J Clin Pathol. 1995;48(9):876-878.

21. Dalerba P, Sahoo D, Paik S, et al. CDX2 as a prognostic biomarker in stage II and stage III colon cancer. N Engl J Med. 2016;374(3):211-222.

22. Guo JT, Ding LH, Liang CY, Zhou NK, Ye QN. [Expression of EYA2 in non-small cell lang cancer]. Zhonghua Zhong Liu Za Zhi. 2009; 31(7):528-531.

23. Fu J, Xu X, Kang L, et al. miR-30a suppresses breast cancer cell proliferation and migration by targeting Eya2. Biochem Biophys Res Commun. 2014;445(2):314-319.

24. Kumar A, Kennecke HF, Renouf DJ, et al. Adjuvant chemotherapy use and outcomes of patients with high-risk versus low-risk stage II colon cancer. Cancer. 2015;121(4):527-534.

25. O'Connor ES, Greenblatt DY, LoConte NK, et al. Adjuvant chemotherapy for stage II colon cancer with poor prognostic features. J Clin Oncol. 2011;29(25):3381-3388

26. Liu Y, Long YH, Wang SQ, Li YF, Zhang JH. Phosphorylation of H2A. XTyr39 positively regulates DNA damage response and is linked to cancer progression. FEBS J. 2016;283(24):4462-4473.

27. Yang AD, Fan F, Camp ER, et al. Chronic oxaliplatin resistance induces epithelial-to-mesenchymal transition in colorectal cancer cell lines. Clin Cancer Res. 2006;12(14 pt 1):4147-4153.

28. Ma JL, Zeng S, Zhang Y, Deng GL, Shen H. Epithelial-mesenchymal transition plays a critical role in drug resistance of hepatocellular carcinoma cells to oxaliplatin. Tumour Biol. 2016;37(5):6177-6184. 


\section{Supplementary materials}

\section{TCGA (GSE62294)}
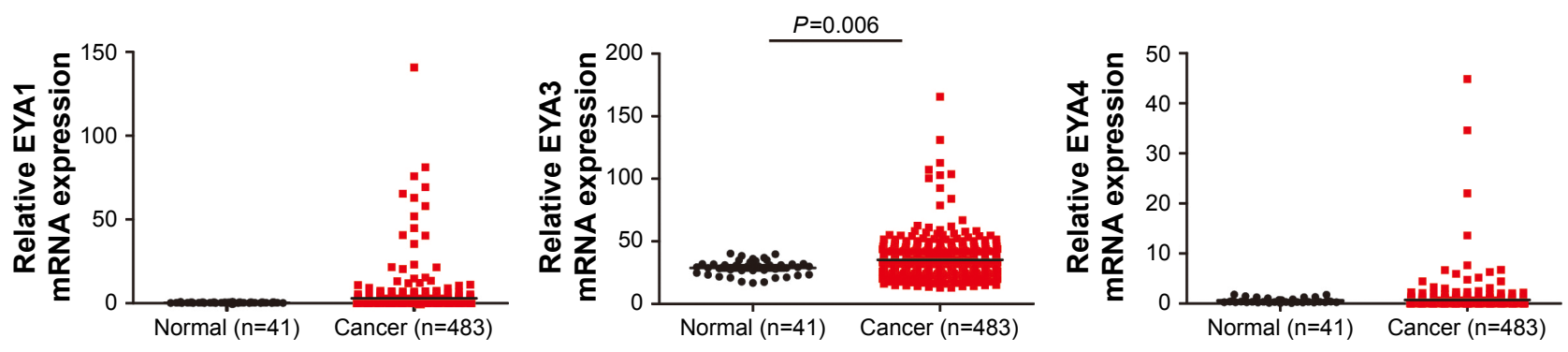

\section{GSE37364}
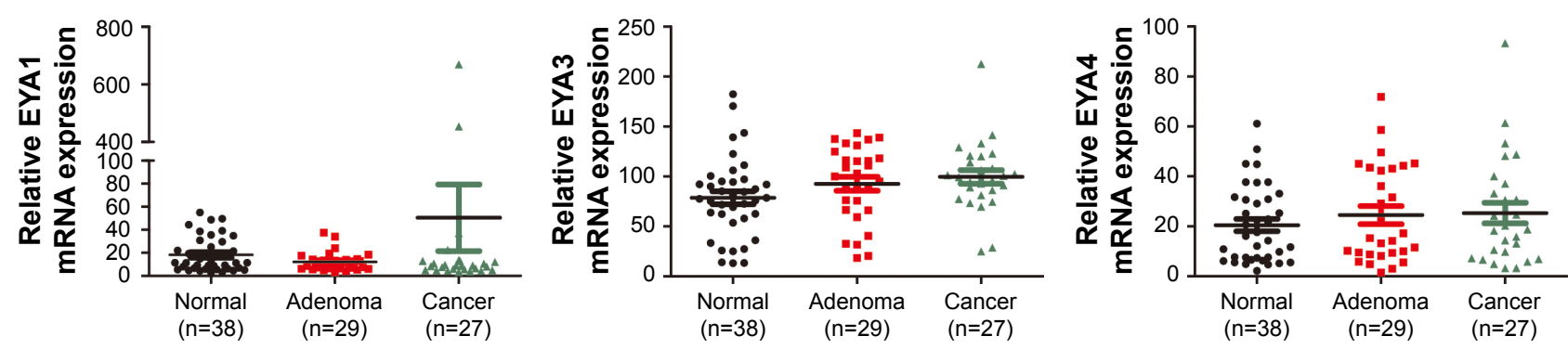

\section{GSE41258}
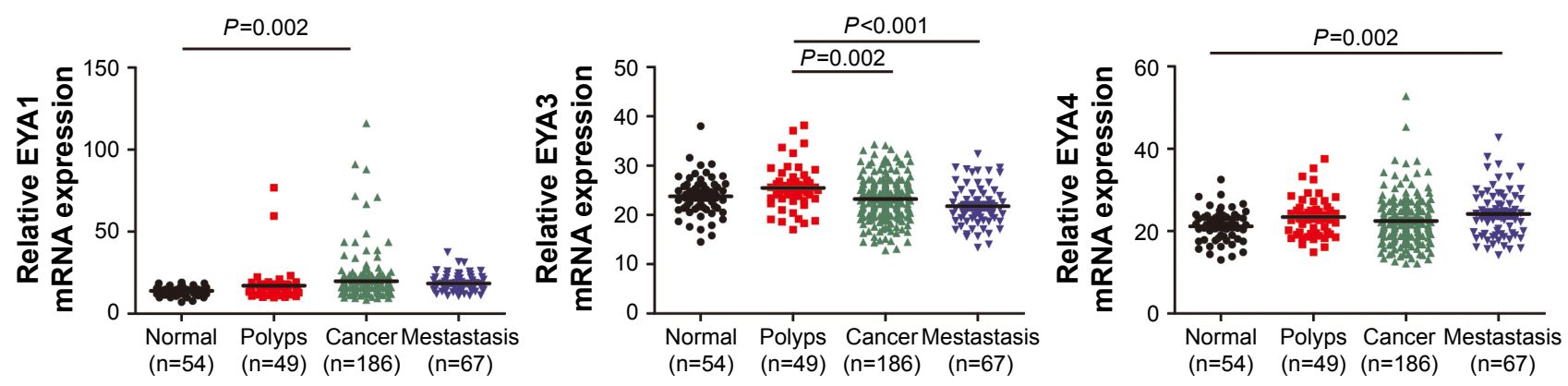

Figure SI Bioinformatics analyses of EYAI, EYA3, and EYA4 mRNA expressions between cancer and cancer-related specimens in three datasets downloaded from GEO database.

Abbreviations: EYA2, eyes absent homologue 2; GEO, Gene Expression Omnibus; TCGA, The Cancer Genome Atlas.

Table SI The clinicopathological features for the patients at stages II and III with or without chemotherapy

\begin{tabular}{|c|c|c|c|c|c|c|}
\hline \multirow[t]{2}{*}{ Factors } & \multicolumn{3}{|l|}{ Stage II } & \multicolumn{3}{|l|}{ Stage III } \\
\hline & Chemotherapy & No chemotherapy & $P$-value & Chemotherapy & No chemotherapy & $P$-value \\
\hline T stage & & & $<0.001$ & & & 0.753 \\
\hline T3 & $192(5 \mid .5)$ & $74(85.1)$ & & $192(64.0)$ & $9(60.0)$ & \\
\hline $\mathrm{T} 4$ & |8| (48.5) & $13(14.9)$ & & $108(36.0)$ & $6(40.0)$ & \\
\hline Resected lymph nodes & & & 0.105 & & & 0.028 \\
\hline$<12$ & $95(25.5)$ & $15(17.2)$ & & $70(23.3)$ & $0(0)$ & \\
\hline$\geq 12$ & $278(74.5)$ & $72(82.8)$ & & $230(76.7)$ & $15(100)$ & \\
\hline Grade & & & 0.827 & & & 0.145 \\
\hline Poor and moderate & $326(87.4)$ & 77 (88.5) & & $24 \mid(80.3)$ & $15(100)$ & \\
\hline Well & $39(10.5)$ & $10(11.5)$ & & $5 I(17.0)$ & $0(0)$ & \\
\hline Missing & $8(2.1)$ & $0(0)$ & & $8(2.7)$ & $0(0)$ & \\
\hline Serum CEA & & & 0.118 & & & 0.704 \\
\hline$<5 \mathrm{ng} / \mathrm{mL}$ & $228(6 I . I)$ & $61(70.1)$ & & $165(55.0)$ & $9(60.0)$ & \\
\hline$\geq 5 \mathrm{ng} / \mathrm{mL}$ & | 45 (38.9) & $26(29.9)$ & & I $35(45.0)$ & $6(40.0)$ & \\
\hline
\end{tabular}

Abbreviation: CEA, carcinoembryonic antigen. 
OncoTargets and Therapy

\section{Publish your work in this journal}

OncoTargets and Therapy is an international, peer-reviewed, open access journal focusing on the pathological basis of all cancers, potential targets for therapy and treatment protocols employed to improve the management of cancer patients. The journal also focuses on the impact of management programs and new therapeutic agents and protocols on

patient perspectives such as quality of life, adherence and satisfaction. The manuscript management system is completely online and includes a very quick and fair peer-review system, which is all easy to use. Visit http://www.dovepress.com/testimonials.php to read real quotes from published authors.

Submit your manuscript here: http://www.dovepress.com/oncotargets-and-therapy-journal 